\title{
Amplitude and Phase Evolution of Optical Fields Inside Periodic Photonic Structures
}

\author{
E. Flück, M. Hammer, A. M. Otter, J. P. Korterik, L. Kuipers, and N. F. van Hulst
}

\begin{abstract}
Optical amplitude distributions of light inside periodic photonic structures are visualized with subwavelength resolution. In addition, using a phase-sensitive photon scanning tunneling microscope, we simultaneously map the phase evolution of light. Two different structures, which consist of a ridge waveguide containing periodic arrays of nanometer scale features, are investigated. We determine the wavelength dependence of the exponential decay rate inside the periodic arrays. Furthermore, various interference patterns are observed, which we interpret as interference between light reflected by the substrate and light inside the waveguide. The phase information obtained reveals scattering phenomena around the periodic array, which gives rise to phase jumps and phase singularities. Locally around the air rods, we observe an unexpected change in effective refractive index, a possible indication for anomalous dispersion resulting from the periodicity of the array.
\end{abstract}

Index Terms-Microscopy, near-field optics, optical imaging, optical interferometry, optical losses, optical planar waveguides, optical propagation, optical scattering, periodic structures, phase measurement, scanning probe microscopy, standing-wave measurements, wavelength measurement.

\section{INTRODUCTION}

$\mathbf{P}$ HOTONIC CRYSTALS are composites of different periodically varied dielectrics [1]-[4]. The amount of interaction between the crystal and the light field can be characterized by the so-called photonic strength of the crystal [5] that is determined by the refractive index contrast, the volume fraction of the constituent materials, and the symmetry of the crystal. For sufficiently large photonic strength, a gap opens up in the photonic dispersion relation (similar to the bandgap for electrons in an atomic lattice). Optical frequencies located inside the gap are forbidden in the crystal. Tailored local defects in a perfect crystal may result in optical states in the gap. Point defects thus act as high- $Q$ resonators [6], whereas line defects guide the light of the allowed optical state through the crystal [7], [8]. The

Manuscript received August 27, 2002; revised January 23, 2003. This work was supported by the "Stichting voor Fundamenteel Onderzoek der Materie (FOM)," which is financially supported by the "Nederlandse Organisatie voor Wetenschappelijk Onderzoek (NWO)." This research is part of the strategic research orientation on "Advanced Photonic Structures" of the MESA ${ }^{+}$Research Institute.

E. Flück, J. P. Korterik, L. Kuipers, and N. F. van Hulst are with the Applied Optics Group, Department of Applied Physics and MESA ${ }^{+}$Research Institute, University of Twente, $7500 \mathrm{AE}$ Enschede, The Netherlands (e-mail: e.flueck@tn.utwente.nl).

M. Hammer is with the Department of Mathematical Science, University of Twente, 7500 AE Enschede, The Netherlands.

A. M. Otter is with the MESA ${ }^{+}$Research Institute, University of Twente, 7500 AE Enschede, The Netherlands.

Digital Object Identifier 10.1109/JLT.2003.810602 tremendous control over light flow afforded by photonic crystals promises a far-reaching miniaturization of integrated optics [9], [10].

In general, photonic crystals are used in combination with conventional waveguiding techniques. Important issues for photonic crystal devices are therefore not only losses, material inhomogeneities, and high fabrication accuracy, but also the coupling between waveguide element and photonic crystal (minimization of impedance mismatch and matching of mode profiles). The photonic crystal structures are mostly investigated with optical reflection and transmission measurements [11]-[16]. From such measurements, information about propagation of light in the crystal can be deduced. Unfortunately, the techniques are restricted to probe a small fraction of the crystalline directions, mainly the angular range close to the propagation directions of light. As a result, it is not possible to characterize a crystal for all wave propagation directions. Furthermore, such "black-box" methods measure the combined effect of all propagation aspects of the photonic structures. In contrast, measurements of the local optical field distribution allow the investigation of each aspect separately. As the relevant length scale of photonic crystals is of the order of the wavelength of light, an instrument is required to investigate the flow of light with subwavelength optical resolution.

A photon scanning tunneling microscope (PSTM) is a near-field optical microscope [17] that can measure local optical properties with subwavelength resolution. With a near-field probe the evanescent fields above a structure are picked up. In this way, optical details about the flow of light are visualized [18]-[26]. Investigations on photonic crystals with a PSTM therefore complement the results obtained by far-field methods. From the optical intensity distribution, any losses inside the structure can be measured locally without any prior assumptions, e.g., concerning coupling losses. Moreover, scattering due to impedance mismatch can be located. Not only is the optical field distribution of light inside a structure measured, but the topography of the structure itself is mapped simultaneously. This can reveal fabrication imperfections with accuracy well below a micrometer and their influence on the optical properties [27]. We use a heterodyne interferometric PSTM. With this instrument, it is even possible to detect the phase evolution of light inside the structure [28]-[31]. Thus, the effective index of refraction is directly measured and multimode behavior is accessible through a simple Fourier analysis [30].

In this paper, we present phase-sensitive PSTM measurements performed on two different three-dimensional (3-D) 
structures with one-dimensional (1-D) periodicity. From the distribution of the optical amplitude, scattering phenomena around both an air rod array and an array of slits (Bragg grating) in a ridge waveguide are investigated. Inside the periodic structure, a wavelength-dependent decay of the optical field is observed and the loss of the structure is determined. In addition, a wavelength-dependent recovery process of light behind the slit array is observed. The measurement of the phase evolution of light in the structure reveals all the waves in the structure. Inside the periodic air rod array region, a local variation in the phase fronts is observed. Behind the air rod structure we observe a network of phase singularities.

Section II gives the experimental details of the investigation. In Section III the measurement of the optical intensity distributions are discussed and compared with simulations. The phase-sensitive experiments are presented in Section IV. The conclusions of our study are given in Section V.

\section{EXPERIMENT}

In a conventional PSTM, a sharp tapered single-mode optical fiber probe is raster-scanned along the surface of the structure under investigation. The fiber probe is kept at a constant height with a tuning fork based shear-force feedback [32]. This height feedback yields the topographical information of the structure. For a tip to surface separation of roughly $10 \mathrm{~nm}$, the evanescent tail of optical fields in the structure is frustrated in the taper region of the fiber probe. As a result, the light is converted into a propagating mode inside the fiber, which is subsequently detected. From the detected intensity as a function of position of the tip, a map of the optical intensity distribution inside the structure is constructed. Already in 1993, Vaez Iravani et al. [33] used heterodyne detection in a near-field optical microscope operated in transmission mode to detect phase effects. Adopting a similar concept, we incorporated a conventional PSTM into one branch of a Mach-Zehnder interferometer to detect the phase of light propagating inside a structure. To enable heterodyne detection, the optical frequency of light in the reference branch is shifted by $40 \mathrm{kHz}$ with an acoustooptic modulator. With a Lock-in Amplifier, a signal is obtained that is proportional to the product of the optical amplitudes in each branch multiplied by the cosine (or sine) of the optical phase difference between the two branches. In a measurement, the length of the reference branch is kept constant, whereas the length of the measurement branch changes due to the raster scan of the tip over the sample. As a result, interference fringes are observed as the tip position changes. From the fringes, the phase evolution of the light inside the structure is visualized as it propagates: in the propagation direction, the spacing of the fringes corresponds to the wavelength of light in the structure [30].

The two branches of the Mach-Zehnder interferometer are reasonably long (1 $\mathrm{m}$ each). A box around the setup strongly reduces thermal drift and airflow that would otherwise disturb the sensitive interferometric measurement. In addition, the stability of the setup is checked before and after each measurement. The experiments are performed with different laser sources: an $\mathrm{HeNe}$ laser, an $\mathrm{Ar}^{+} / \mathrm{Kr}^{+}$laser, and a tuneable frequency-doubled (Ti: Sapphire-pumped) optical parametric oscillator. Light is coupled into the photonic structure using a microscope objective $(0.4 \mathrm{NA}, 20 \times)$.

Near-field aperture probes are fabricated by local heating and simultaneous pulling of a single-mode optical fiber. In this way, a sharp taper and an end-face of a diameter $\sim 80 \mathrm{~nm}$ are produced. Subsequent coating with metal and side-on milling using a focused ion beam reproducibly yields circular apertures with a controllable diameter [34]. In all measurements discussed in this paper, coated fiber probes with aperture diameters of $80 \mathrm{~nm}$ were used. Previous investigations on strongly scattering structures showed that scattered light emanating from the structure can couple directly into the fiber probe [35] swamping the signal originating from the light propagating inside the structure. Local probing of field intensities with uncoated fiber probes therefore becomes impossible. Coated fiber probes reduce the pick up of scattered light significantly. The coating also prevents the detection of light that travels along and just above the sample surface. We have found no influences on the optical properties caused by the probe with its metal coating, in agreement with theoretical calculations that predict a very small effect [36].

The basis for our structures is an $\mathrm{Si}_{3} \mathrm{~N}_{4}$ ridge waveguide, grown on top of a 3.2- $\mu \mathrm{m} \mathrm{SiO}_{2}$ layer on a Si-substrate. The height and width of the waveguide are $55 \mathrm{~nm}$ and $1.5 \mu \mathrm{m}$, respectively. The ridge height is $11 \mathrm{~nm}$, thus a slab layer of $44 \mathrm{~nm}$ surrounds the waveguide. In the interesting wavelength range between 585 and $647 \mathrm{~nm}$ (vacuum wavelength), this geometry allows only the propagation of the fundamental transverse electric mode $\left(\mathrm{TE}_{00}\right)$ and no transverse magnetic modes (TM). A periodic pattern is milled into the waveguide ridge with a beam of gallium ions that is focused to a small spot (diameter $\sim 8 \mathrm{~nm}$ ). The milling allows individual fabrication of air rods and slits into the waveguide ridge. The two different photonic structures fabricated and investigated are a ridge waveguide with 15 air rods and a ridge waveguide with an array of 15 slits. Both arrays are designed with a periodicity of $220 \mathrm{~nm}$. The diameter of the air rods is $110 \mathrm{~nm}$. The slits are $110 \mathrm{~nm}$ wide and $2.5 \mu \mathrm{m}$ long. In both cases, the milling was performed such that the feature depth is approximately $70 \mathrm{~nm}$. This means that the high-index $\mathrm{Si}_{3} \mathrm{~N}_{4}$ layer of $55 \mathrm{~nm}$ is penetrated completely. The accuracy of the periodicity is restricted to $\sim 6 \mathrm{~nm}$ by the steering accuracy of the ion beam, whereas the milling accuracy is given by the beam size of $\sim 8 \mathrm{~nm}$.

\section{OPTICAL INTENSITY DISTRIBUTIONS OF LIGHT}

\section{A. Measurements}

PSTM images of the two different waveguide structures are presented in Fig. 1. The topography of both structures is shown in Fig. 1(a) and (c) and the optical intensity maps are given in Fig. 1(b) and (d). In both measurements, light is coupled into the waveguide (incoupling position is at a large distance of $4 \mathrm{~mm}$ from measurement area) and propagates from top to bottom in the images. For the measurement on the air rod structure [Fig. 1(b)], light with a wavelength of $\lambda_{0}=647 \mathrm{~nm}$ was coupled into the waveguide, whereas for the measurement on the slits [Fig. 1(d)], a wavelength of $610 \mathrm{~nm}$ was used.

In the topographical information of the structures, the 11-nm-high waveguide ridges are easily seen. The two different 

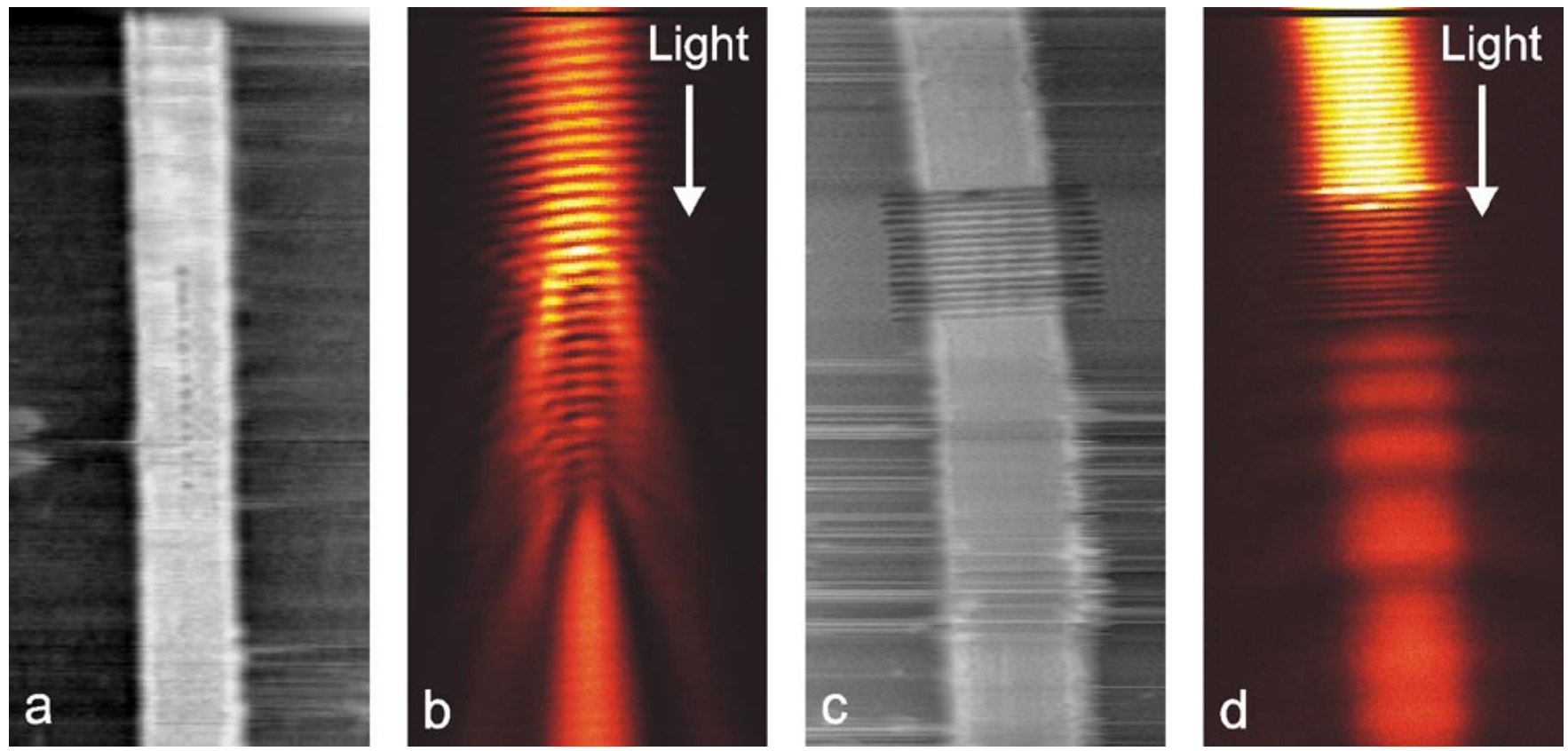

Fig. 1. Conventional PSTM measurement performed on two different structures: a channel waveguide containing 15 air rods-(a) topography and (b) optical intensity distribution, and a channel waveguide containing 15 slits-(c) topography and (d) optical intensity distribution. The periodicity of the arrays is $220 \mathrm{~nm}$, and the diameter of the holes, as well as width of the slits, is $110 \mathrm{~nm}$. The width of the slits is $2.5 \mu \mathrm{m}$. Light propagates from top to bottom (arrow). Image size: (a) and (b) $5.8 \mu \mathrm{m} \times 11.1 \mu \mathrm{m}$ (horizontal $\times$ vertical); (c) and (d) $4.0 \mu \mathrm{m} \times 18.3 \mu \mathrm{m}$ (horizontal $\times$ vertical).

TABLE I

Values Waveguide and Structure Dimension Obtained From the Measurements Shown in Fig. 1. Comparison With the Fabrication Values Shows a SLight DifFerence With the Measured Values

\begin{tabular}{c|c|c|c}
\hline & Fig. 1a (rods) & Fig. 1c (slits) & Fabrication values \\
\hline Width of the waveguide: & $1558(33) \mathrm{nm}$ & $1627(13) \mathrm{nm}$ & $1500 \mathrm{~nm}$ \\
\hline Diameter of the air rods: & $111(37) \mathrm{nm}$ & & $80 \mathrm{~nm}$ \\
\hline Dimension of the slits: & & $2333(13) \mathrm{nm} \times 122(30) \mathrm{nm}$ & $2500 \mathrm{~nm} \times 80 \mathrm{~nm}$ \\
\hline
\end{tabular}

structures produced in the ridge with a focused ion beam [the array of 15 air rods in Fig. 1(a) and the array of 15 slits in Fig. 1(c)] are clearly resolved. From the topographical images, we measure the actual waveguide and array dimensions given in Table I. The measured sizes of the subwavelength features (Table I) differ from the fabrication values. We attribute the broadening of the features to the fabrication process itself. In particular, charging of the material $\left(\mathrm{Si}_{3} \mathrm{~N}_{4}\right)$ can result in enlargement of the feature sizes.

In both topographical images, horizontal stripes are visible. In the lower part of Fig. 1(c), some of those stripes are more pronounced. These are fluctuations in the interaction between tip and sample caused by changes in the environment, which make the feedback mechanism less stable. Since the tip scans horizontally, instability induces stripes in this direction. It is important to realize that these fluctuations in height $(7 \mathrm{~nm}$ at most) have no significant influence on the simultaneously recorded optical image [see Fig. 1(d)].

Fig. 1(b) and (d) depicts the measured optical intensity distribution of light inside the structure. In front of the periodic array of air rods and slits, horizontal intensity stripes are clearly resolved in both images. A perfect periodicity over a large range in front of the structures is found. Analyzing this periodic pattern by using Fourier transformation, we find for Fig. 1(b) a periodicity of 208(3) nm and for Fig. 1(d) a periodicity of 223(3) $\mathrm{nm}$. Obviously, this subwavelength periodicity indicates that the pattern is caused by interference: reflected and incoming light built up a standing wave in front of the periodic structures. For the different wavelengths of light coupled in, different periodicities are found. From the periodicity of the standing wave, one can calculate the wavelength of the contributing modes. Table II shows an overview of measurements performed for different wavelengths. For comparison, we calculated the wavelength of the waveguide modes using an effective index of refraction of the waveguide, which depends on the vacuum wavelength of light and on the geometry of the waveguide. This index of refraction is calculated by the effective index method [37]. Vice versa, we can determine the effective index of refraction from the measurements: $\mathrm{n}_{\text {eff }}=\lambda_{\text {mode }} / \lambda_{0}$. As can be seen in Table II, good agreement between the calculated and measured values is found.

Just in front of the array structure, the standing waves of the two images differ in shape. In the case of the slits [Fig. 1(d)], the horizontal stripes are straight, whereas in front of the air rod structure [Fig. 1(b)], a slight curvature of these stripes is observed. This observation may be explained in a simple picture. In the case of the air rod structure, it seems that the pattern is built up from interference between circularly scattered waves and incoming plane waves, which leads to a parabolic curvature in the first few intensity stripes [17]. In the waveguide with the 
TABLE II

Determination OF THE WaVElENGTH OF Light Propagating IN THE WAVEGUIDE. First COLUMN: THE VACUUM WAVELENGTH $\lambda_{0}$ OF Light. SECOND Column: Calculated WaVElength $\left(\lambda_{0} / \mathrm{n}_{\text {eff }}\right)$ OF THE TE $\mathrm{E}_{00}$ WaVEGuide Modes. To ObTaIn These Values, THE EFFective IndeX METHOD Was USED TO CALCULATE THE EFFECTIVE REFRACTIVE INDEX $\mathrm{n}_{\text {eff }}$ OF THE Structure. From the Periodicity OF THE Standing WAVES, THE VALUES OF THE WAVELENGTH IN THE WaVEGUides ARE DETERMINED FOR BOTH THE AIR ROD AND SLIT STRUCTURE AND ARE GIVEN IN THE THIRD AND FOURTH COLUMN

\begin{tabular}{c|c|c|c}
\hline vacuum wavelength & calculated & \multicolumn{2}{|c}{ experimental } \\
$\lambda_{0}$ & $\lambda$ TE $_{00}$ mode & $\lambda_{\text {mode }}$ air rods & $\lambda_{\text {mode }}$ slits \\
\hline $585 \mathrm{~nm}$ & $399 \mathrm{~nm}$ & $398(5) \mathrm{nm}$ & $406(4) \mathrm{nm}$ \\
$600 \mathrm{~nm}$ & $410 \mathrm{~nm}$ & $405(3) \mathrm{nm}$ & $407(6) \mathrm{nm}$ \\
$610 \mathrm{~nm}$ & $417 \mathrm{~nm}$ & $416(4) \mathrm{nm}$ & $426(7) \mathrm{nm}$ \\
$633 \mathrm{~nm}$ & $433 \mathrm{~nm}$ & $428(7) \mathrm{nm}$ & $439(8) \mathrm{nm}$ \\
$647 \mathrm{~nm}$ & $443 \mathrm{~nm}$ & $441(2) \mathrm{nm}$ & $446(3) \mathrm{nm}$ \\
\hline
\end{tabular}

slits structure, light is reflected into a plane wave, resulting in straight interference stripes.

Inside the air rod and the slit array a periodic pattern is still visible, but the absolute optical intensity decreases inside both structures. Around the 15 air rods [Fig. 1(b)], it can be seen that light passes on the left and right side along the air rod array. Beyond the final air rod, light is recovered in the fundamental mode of the unperturbed waveguide. Fig. 1(b) shows that the mode almost completely recovers after several hundred nanometers beyond the final air rod. A different recovery process is observed after the 15-slit structure [Fig. 1(d)]. Intensity minima and maxima occur in a nonperiodic way. Interestingly, with increasing distance beyond the slit array, the distance between two minima increases. This unexpected phenomenon will be further analyzed in Section III-C.

To analyze data quantitatively, line traces taken parallel to the waveguide axis are investigated. Several adjacent line traces around the center of the waveguide are summed up to achieve a better signal-to-noise ratio (SNR). Fig. 2(a) shows the resulting average intensity produced by summing up line traces of a 120-nm-wide area in the center of the waveguide of the measurement in Fig. 1(b). In a similar way, Fig. 2(b) has been made by summing up line traces over a $1-\mu \mathrm{m}$-wide area from Fig. 1(d). In both figures, the propagation direction of the light is from the left to the right. The location of the periodic array is indicated by the dashed square.

In the left part of Fig. 2(a) and (b), the periodic oscillations of the standing wave (in front of the periodic arrays) are visible. As discussed previously and shown in Table II, the pattern has a periodicity of $\lambda_{0} /\left(2 \mathrm{n}_{\mathrm{eff}}\right)=\lambda_{\text {mode }} / 2$, half the wavelength of light in the waveguide. A difference in the intensity profile of the standing wave in front of the air rods and slits is clear. In front of the 15 air rods [Fig. 2(a)], a continuous attenuation of both intensity and the modulation depth is observed over a range of $1 \mu \mathrm{m}$. Further away from the 15 air rods, as well as in front of the 15 slits, the intensity and modulation depth of the standing wave are constant. These different observations on the two structures hold for all wavelengths of light. The decrease in intensity and modulation depth indicates a loss of coherence of the standing wave. We find a decay of intensity in front of the 15 air rods of $1.05 \mu \mathrm{m}^{-1}=4.1 \mathrm{~dB} / \mu \mathrm{m}$.
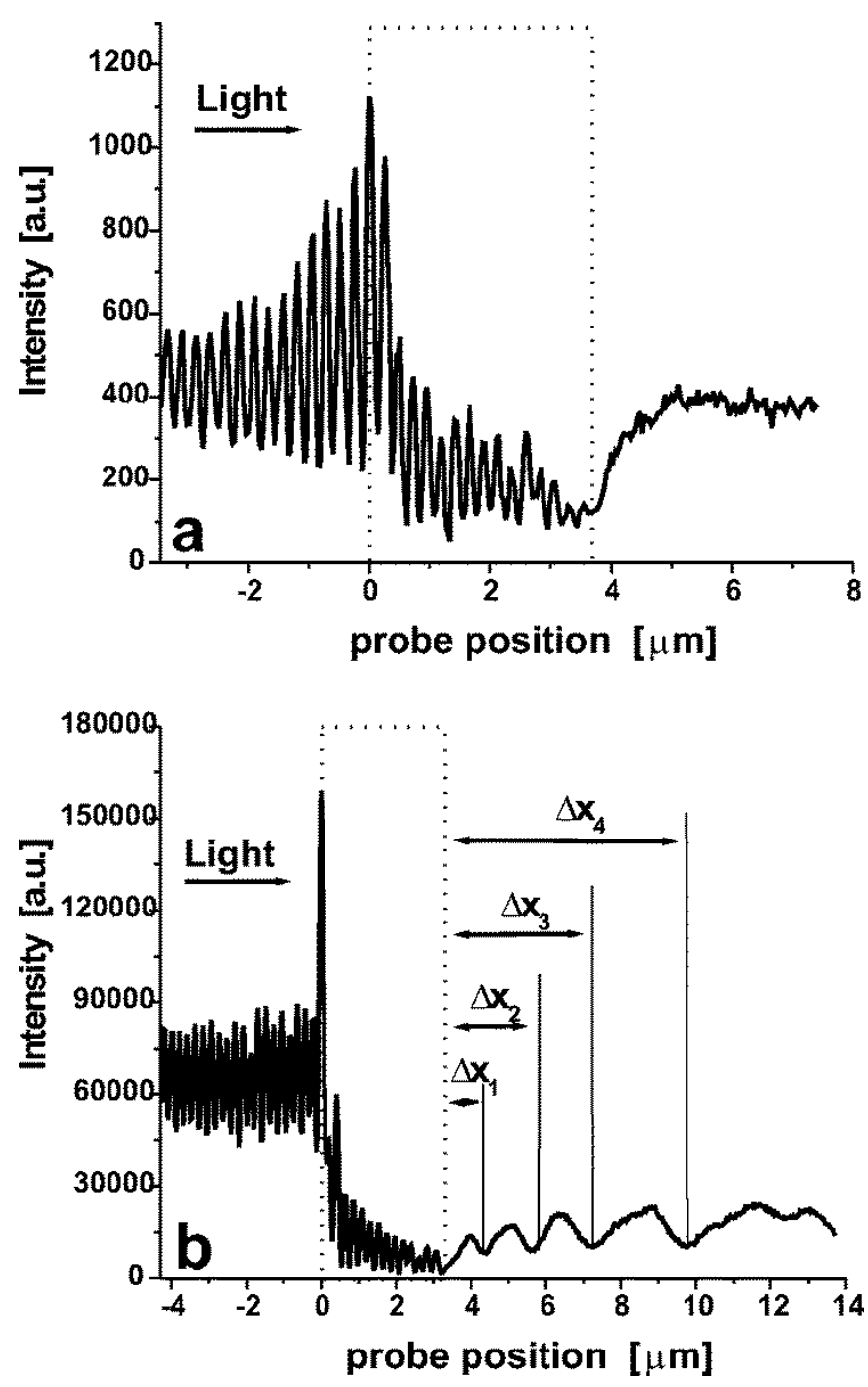

Fig. 2. Line traces obtained from the measurements in Fig. 1. To achieve a better signal to noise ratio, several adjacent line traces were summed up to produce the graphs shown here. Light propagates from left to right. The dashed square gives the location of the (a) 15 air rods and (b) 15 slits, respectively.

Loss of coherence can be caused by a decrease of the amplitude of the reflected light with distance, a number of different $\mathrm{k} / /$-vectors (parallel to the $\mathrm{Si}_{3} \mathrm{~N}_{4}$ slab plane) being present in the reflected light or a combination thereof. Clearly, the amplitude of a circularly scattered wave will decrease with distance from the structure. However, this decrease is too slow (several $\mu \mathrm{m}^{-1}$ ) to explain the decrease of the modulation depth in front of the 15 air rods. Excitation of "leaky modes" can lead to a number of different discrete $\mathrm{k}_{/ / \text {-vectors being present in the re- }}$ flected light. "Leaky modes" are high loss modes, which are not supported by the waveguide geometry [35]. If scattering of light at the air rods excites leaky modes, an interference pattern with a fast exponential decay in front of the air rods is expected. However, the exponential decay (in the order of $100 \mathrm{~nm}^{-1}$ ) is faster than $1.05 \mu \mathrm{m}^{-1}$. We therefore attribute the loss of coherence to a combination of the excitation of "leaky mode" and the circularly scattered waves. In the case of 15 slits, no change in the modulation depth of the standing wave is observed. The amount 
of scattering light into "leaky modes" and build up of circularly scattered waves is significantly reduced with respect to the air rods as a result of the straight geometry.

In front of both periodic structures, a high intense peak is present. The peak intensity compared to the amount of incoming light can be up to $2.5 \times$ in the case of the 15 slits and $1.6 \times$ for the air rod structure. We attribute this high intensity of the first peak to light that is directly scattered out of the slit into the aperture probes due to the impedance mismatch between the ridge waveguide and the periodic structure.

In the region of the photonic structure (dashed square), a periodic intensity pattern is found as well. There are 15 peaks located inside the dashed square that are found in both cases [Fig. 2(a) and 2(b)]. The periodic distance between those peaks corresponds to the periodicity of the array $(220 \mathrm{~nm})$. The difference in periodicity of the standing wave and the interference pattern inside the structure cannot be seen by eye from Fig. 2. To resolve them, a Fourier transformation has been performed. We suggest that direct probing of so-called Bloch modes takes place. The nodes result directly from the periodicity of the structure. The observed modes do exhibit a periodic intensity pattern with the periodicity of the structure for every optical frequency. Topographical artifacts in the optical image can be excluded for two reasons. First, the exponential decay observed for the maxima of intensity inside the array does not reproduce for the minima of intensity in the array. In the case of artifacts, one would expect to observe for both, maxima and minima, the same decay. Second, the intensity stripes found in the periodic region of the air rods are elongated in the horizontal direction. An artifact induced by topography would result in a symmetric pattern with a dimension corresponding to the probe size convoluted with the air rod diameter. Our apertures are circularly symmetric, so that an elongation in only one direction is not possible.

Behind the periodic structures, light recovers differently for both structures. Some hundreds of nanometers behind the last air rod [Fig. 2(a)], the fundamental mode $\left(\mathrm{TE}_{00}\right)$ of the waveguide is recovered. The recovery of light behind the slits is different compared with the air rods (as discussed previously). Clearly, a nonperiodic intensity pattern is visible in Fig. 2(b).

\section{B. Simulations}

The field around the periodic structures has been calculated as a function of wavelength to allow direct comparison to the PSTM data. The 2-D simulations are based on Maxwell's equations in the frequency domain. The interesting region of the structure is enclosed by artificial boundaries, which are oriented parallel to the dominant direction of light propagation and located such that all guided fields in the structure are negligible at the boundary positions. This leads to a discrimination of the mode spectra on the waveguide segments; the mode sets become numerically manageable. Separately on each segment, the electric field (E-field) is written as a mode superposition where, depending on the investigated structure forward and backward traveling, propagating and evanescent terms are included. Bidirectional projection of the adjacent fields at the junctions then

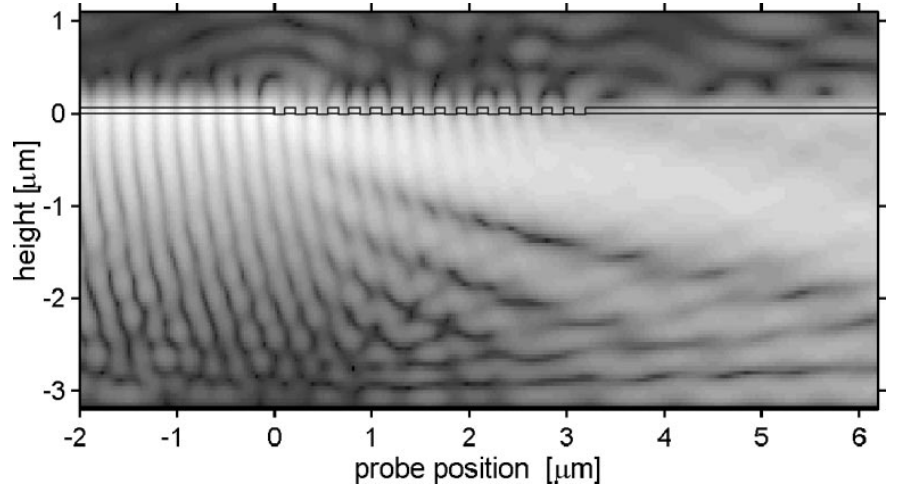

Fig. 3. $\left|E_{\perp}\right|^{2}$ distribution of a 2-D simulation of a cross section through a waveguide ridge containing 15 slits is shown. The incoming light propagates from left to right. On the left side, a standing wave in front of the slit array is visible. It is built up by interference between incoming and reflected light by the slits. Furthermore, light scatters from the first slit into the substrate. An interference pattern is produced between propagating light in the guiding layer and reflected light from the $\mathrm{Si}$-substrate.

allow the setup of a linear system of equations for the coefficients in the mode superposition. See [38] and [39] for details on the simulation technique.

Fig. 3 shows the simulation of the $\left|E_{\perp}\right|^{2}$ distribution for a cross section through a waveguide containing 15 slits. Light $\left(\lambda_{0}=632.8 \mathrm{~nm}\right)$ is launched from the left side and propagates to the right side. In our PSTM measurements, TE polarized light was coupled into the structure. As a result, we compare the measurement to only one component of the simulated E-field, the one perpendicular to the simulation plane shown in Fig. 3. A logarithmic gray scale gives the square of this E-field component $\left(\left|\mathrm{E}_{\perp}\right|^{2}\right)$, where bright means high intensity and dark represents low intensity. The guiding $\mathrm{Si}_{3} \mathrm{~N}_{4}(\mathrm{n}=2.01)$ waveguide layer is $55 \mathrm{~nm}$ thick and is deposited onto a $3.2-\mu \mathrm{m}$-thick layer $\mathrm{SiO}_{2}(\mathrm{n}=1.45)$. The $\mathrm{SiO}_{2}$ is grown on a standard $\mathrm{Si}$-substrate. The $\mathrm{SiO}_{2}-\mathrm{Si}$ interface has an appreciable reflection coefficient for many wavelengths of light. For this reason, a perfect mirror is chosen to resemble the physical buffer-substrate interface in the simulations, which is realized by placing the artificial computational window boundary at this position. The horizontal axis in Fig. 3 is the relative probe position with respect to the first slit. In front of the slit array, the standing wave pattern is clearly present. At the position of the first slit, light is scattered mainly back into the waveguide and into the $\mathrm{SiO}_{2}$ substrate. The light scattered into the substrate gets reflected at the Si-substrate located at $-3.2 \mu \mathrm{m}$. As a result, the interference of guided light with scattered light takes place behind the slit structure.

To compare the simulations directly with the measurements, we calculated $\left|\mathrm{E}_{\perp}\right|^{2}$ for a line located $10 \mathrm{~nm}$ above the waveguide (Fig. 4). Again, the standing wave in front of the slit array (indicated by the dashed square) is clear. At the location of the first slit, the highest intensity of the distribution is found. Inside the array, a decay in intensity is observed. Behind the 15 slits, a nonperiodic recovery of light in the waveguide is found. Two pronounced minima are found in the calculation window (marked by $\mathrm{x}_{1}$ and $\mathrm{x}_{2}$ ). Different simulations show that this nonperiodic pattern beyond the slits changes as a function of the position of the Si-substrate, e.g., if the Si-substrate is located $10 \mu \mathrm{m}$ underneath the waveguiding layer, the nonperiodic interference pattern disappears. 


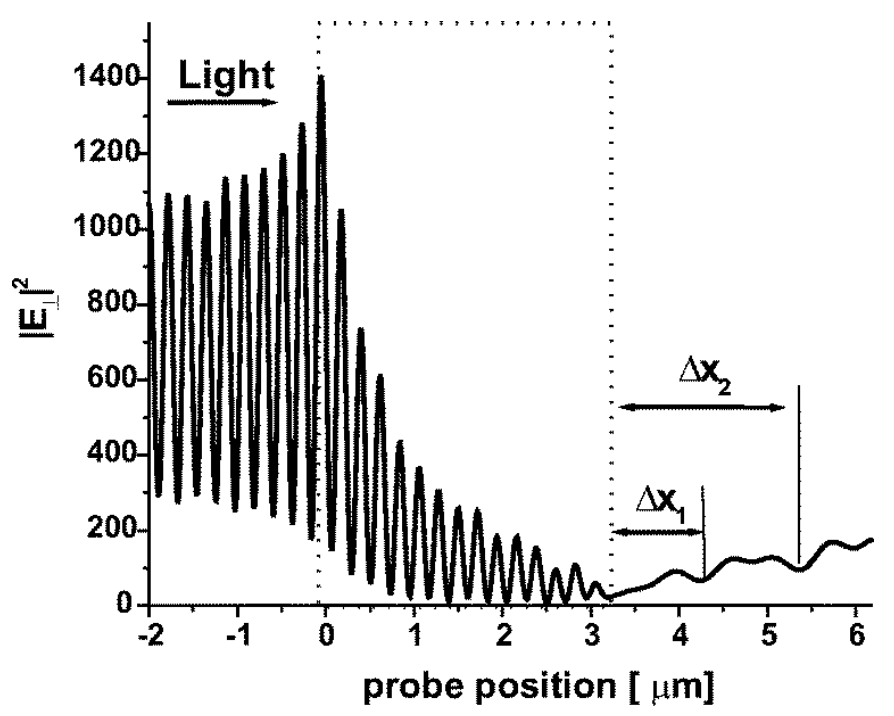

Fig. 4. Line trace of intensity taken from the simulation at a horizontal position $10 \mathrm{~nm}$ above the waveguide. Besides the standing wave, the exponential decay of intensity inside the array is depicted. After the slits, a nonperiodic recovery is found.

\section{Comparison and Discussion}

Both measurements and simulations were performed for a range of different wavelengths: 585, 600,610, 633, and $647 \mathrm{~nm}$. In all line traces [Figs. 2(a) and (b) and 4], light propagates from left to right and builds up a standing wave in front of the array. The standing wave periodicity fits perfectly with $\lambda_{0} /\left(2 \mathrm{n}_{\mathrm{eff}}\right)=$ $\lambda_{\text {mode }} / 2$. Furthermore, the first air rod and slit always produce the most intense peak of the overall field distribution. The modulation depth of the standing wave in the simulation changes in a similar way as in the measurements of the 15 air rods.

The period of the interference pattern inside the periodic structures is found to be $220 \mathrm{~nm}$ for all wavelengths and for both the measurements as well as for the simulations. Since the array consists of 15 periods, 15 intensity maxima located in the structure are found. For different line traces measured for different wavelength $\lambda_{0}$, we fitted an exponential decay $\exp (-\beta x)$ through the maxima of intensity located inside the periodic structure. The decay rate $\beta$ is the free fit parameter and $x$ the probe position with respect to the first air rod or slit.

Fig. 5 depicts the obtained decay rates as a function of $\lambda_{0}$. In the case of the 15 air rods, we fitted an exponential decay through all 15 maxima located in the periodic array. Comparison to the fits obtained with the simulations show good agreement both for the wavelength dependence and for the absolute values. Comparing the measurement on 15 slits (Fig. 2) with the simulation (Fig. 4) shows an apparent discrepancy at the location of the first slit. As explained in Section III-A, a large peak at the first slit is measured and attributed to scattering. A comparable peak is not clearly visible in the simulations. However, the calculated $\left|\mathrm{E}_{\perp}\right|^{2}$ does show a circular pattern indicating a scattered wave originating from the area of the first air rod. Note that this scattered light is picked up more readily by our near-field probe than the evanescent tails of $\left|\mathrm{E}_{\perp}\right|^{2}$ above the rest of the structure. The measured intensity at the front of the structure can therefore be greater (for equal calculated $\left|\mathrm{E}_{\perp}\right|^{2}$ ) than above the rest of the structure, because the relative contribution of scattered light compared with evanescent fields is much larger at that position.
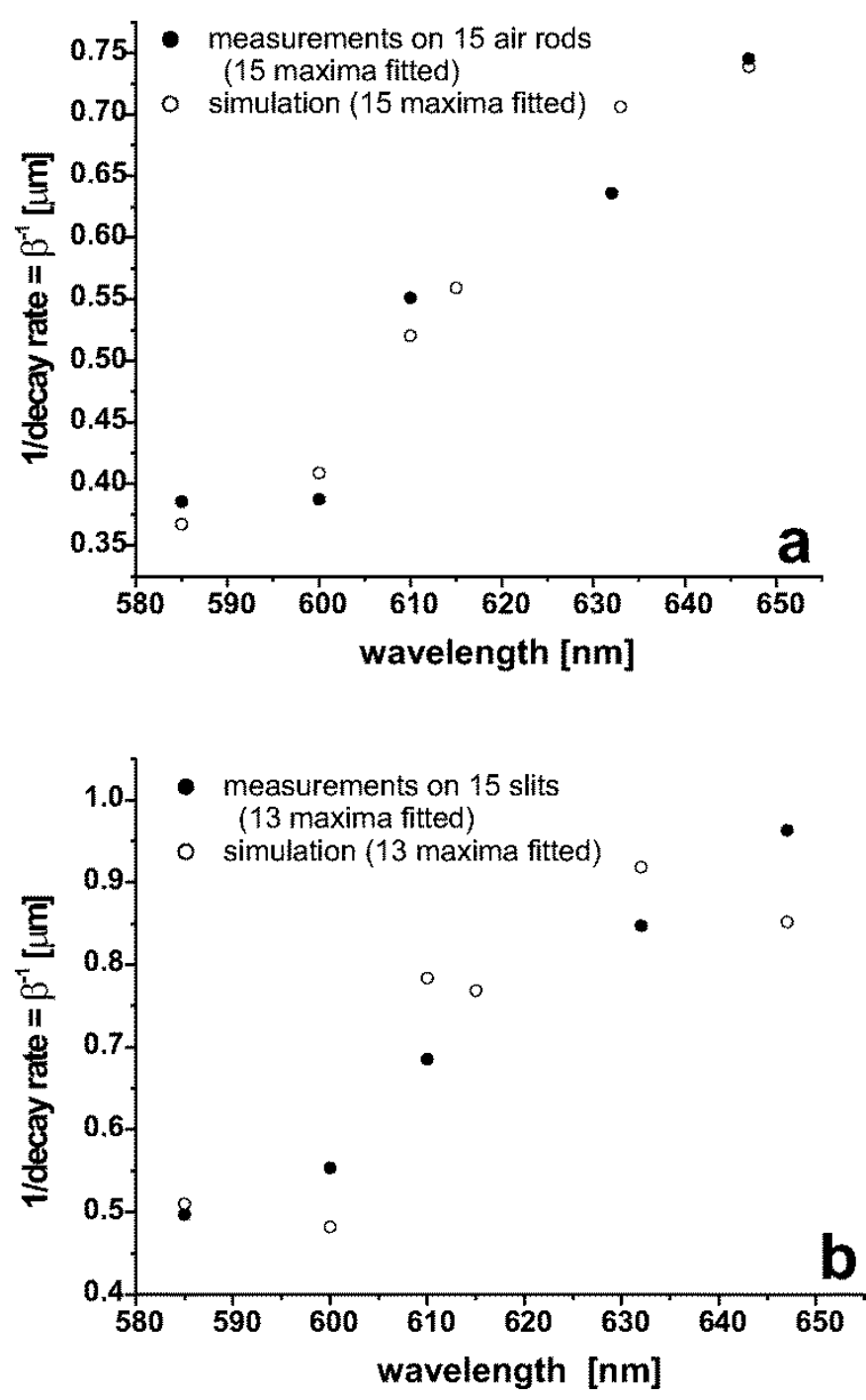

Fig. 5. Decay rates of intensity inside the periodic array determined for different wavelengths of light coupled into the waveguide. A good agreement between measurement and simulation is found for both structures. A fit with $\exp (-\beta \mathrm{x})$ was performed to line traces as shown in Fig. 2(a) and (b). (a) For the air rod array structure. (b) For the slit structure.

For the comparison of the decay inside the structure, we neglect the first and second peak. Thus, 13 maxima have been fitted from the measurements on the slit array. Fig. 5(b) shows the decay rates found, which we again compare with decay rates obtained from the simulations. Here, the decay rates for the simulations have been determined by fitting only 13 peaks. Impedance mismatch occurs when light couples from the last air rod or slit to the unperturbed ridge waveguide again. Because of the very low local intensity the inclusion of the last peak makes no difference to the exponential fit. From the exponential decay rates, we calculate that the periodic structures have losses between 4.4 and $11.4 \mathrm{~dB} / \mu \mathrm{m}$. The decay is faster for shorter wavelength. Scattering phenomena generally increase with decreasing wavelength, where the dependence obeys a power law. We attribute the wavelength-dependent decay found in our periodic arrays to scattering on the air material interface.

As a next step, we investigate the origin of the maxima and minima in the recovery of light beyond the 15 slits. The $\mathrm{Si}$ layer, which lies $3.2 \mu \mathrm{m}$ underneath the $\mathrm{Si}_{3} \mathrm{~N}_{4}$ layer, can be approximated as a mirror, as the simulations showed. There, 


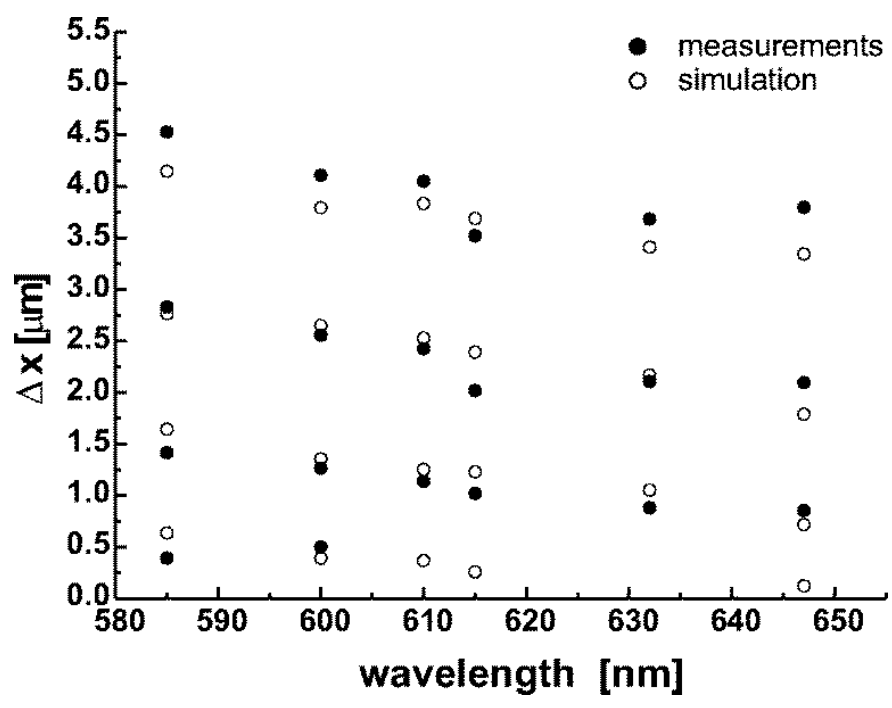

Fig. 6. The recovery of single mode behind the 15 slits involves a nonperiodic intensity pattern. The distance between the minima occurring and the last slit are plotted as a function of wavelength. Good agreement is found between measurement (filled dots) and simulation (open circles).

light scattered into the $\mathrm{SiO}_{2}$ at the first slit gets reflected on the $\mathrm{Si}$-substrate. Interference takes place in the waveguide behind the slits structure with the light that is directly transmitted by the slit structure. In Fig. 6, the distances $\Delta x$ [see Figs. 2(b) and 4] between the minima occurring and the last slit position are plotted as a function of wavelength. We find that for larger wavelength, the minima are located closer to the periodic structure than for shorter wavelength. Good agreement with the simulation is found for the positions of the minima occurring over large ranges behind the slits. As a result, the observation of this nonperiodic interference pattern behind the slits is a measure of the thickness of the underlying $\mathrm{SiO}_{2}$ layer. Normally, layer thicknesses are globally determined by ellipsometry, whereas our measurement tool reveals it locally. In contrast to the slits, the air rod structure scatters a much smaller amount of light to the direction of the Si-substrate and no oscillations in the recovery are found there [see Fig. 2(a)].

\section{Phase Mapping of Light Around Periodic AIR ROD ARRAY}

As discussed in Section II, not only is the optical amplitude measured, but our heterodyne interferometric PSTM also reveals the phase evolution. From the measured signals (amplitude times the cosine of the phase difference and amplitude times the sine of the phase difference), we extract the cosine of the phase only. Fig. 7 shows a map of the cosine of the phase of the light around the 15 air rod structure. The periodic air rod array is located in the center of the image. Light $\left(\lambda_{0}=632.8 \mathrm{~nm}\right)$ enters the structure from the top of the image. A wealth of different phase patterns is observed. The whole image contains all information of contributing modes, which have various wavevectors (different spatial directions and different absolute values), are leaky or scatter out of the confining layer. In the upper half of Fig. 7, the optical wavefronts of the fundamental waveguide mode are visible as flat phase fronts. With a Fourier analysis, the periodicity of the wavefronts is found to be 439(4) nm,, the wavelength of light in the waveguide structure, which immediately yields the effective refractive index for the funda-

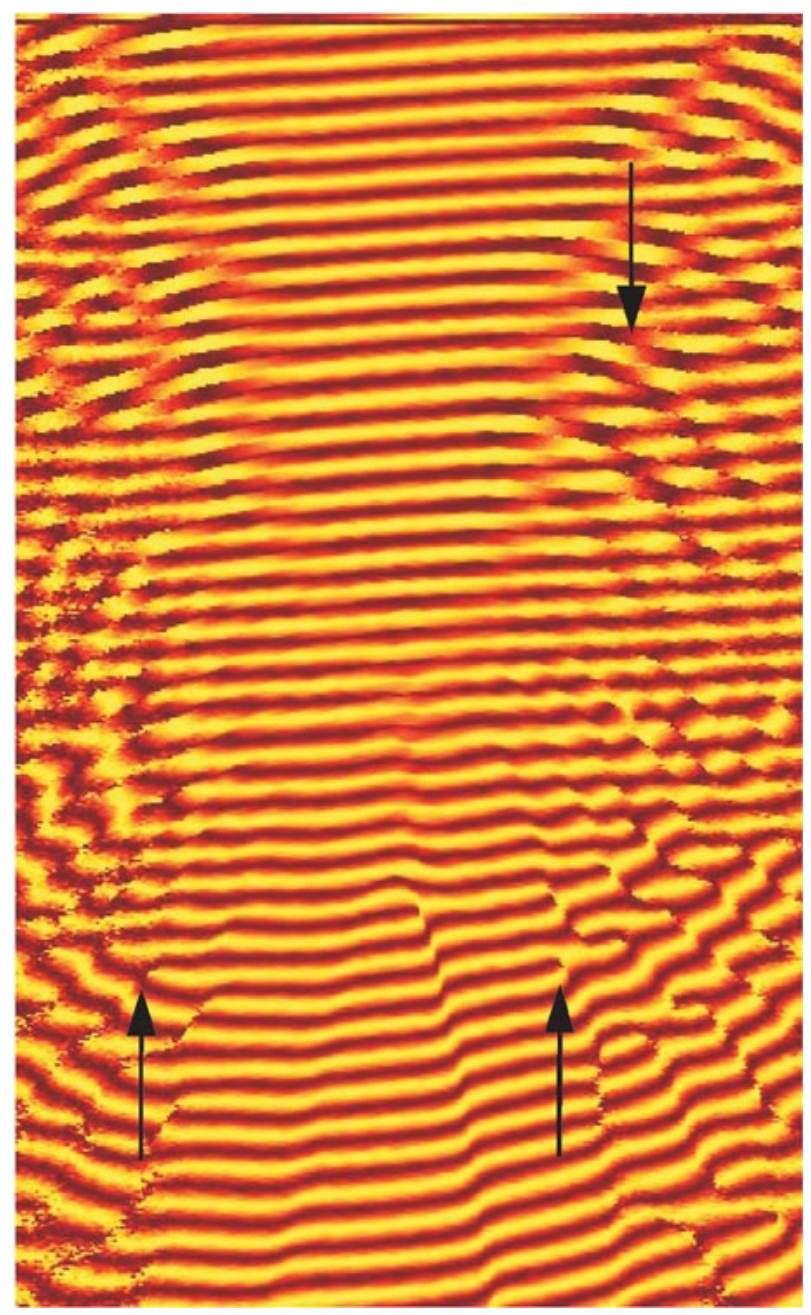

Fig. 7. The phase evolution of light propagating through the waveguide containing 15 air rods is visualized. In this image, the cosine of phase is depicted. The incoming light propagates from top to bottom. We find circularshaped waves, as well as many phase irregularities in the upper and lower part of the image (a number of which are indicated by an arrow). Due to interference between counterpropagating light (upper part) or copropagating light (lower part), phase jumps and phase singularities occur. Image size: $9.33 \mu \mathrm{m} \times 17.23 \mu \mathrm{m}$ (horizontal $\times$ vertical $)$.

mental waveguide mode: $\mathrm{n}_{\mathrm{eff}}=1.441(0.014)$. This value differs slightly from the calculated index of refraction of 1.460 . Given the slightly different geometry of the waveguide compared with the design values (see Table I), this difference in refractive index is easily explained. Fig. 7 also shows a circular wave superimposed on the plane wavefronts. These circular waves have their origin in the center of the image, where the air rods are located. Scattering of incoming light takes place at the air rod array and generation of circular waves takes place. As discussed in Section III, these circular-shaped waves contribute to the change in modulation depth of the standing wave.

Due to interference between the wavefronts of the incoming light and circular waves with origin in the center of the image, different phase anomalies are produced. In the upper part of the image, we find jumps in the phase. They are a result of interference of light that propagates in almost opposite directions (with a crossing angle close to $\pi$ ) and their position changes during a single oscillation of the field. In the lower part of the image, phase singularities are observed. They occur when dif- 

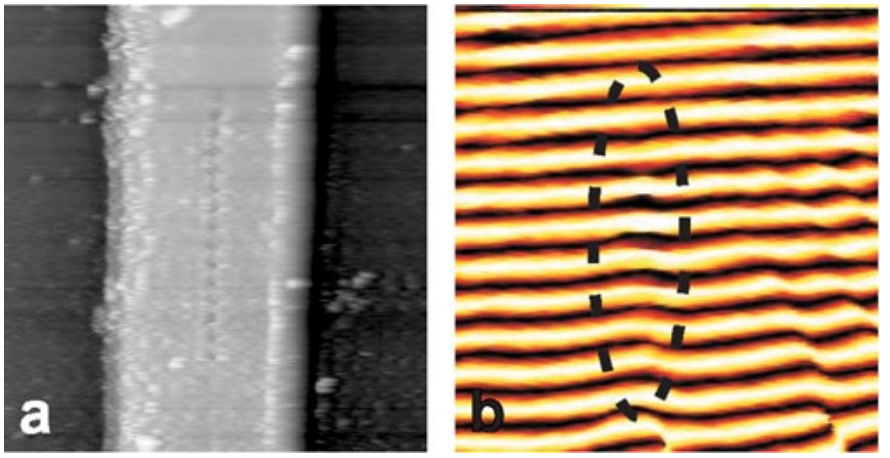

Fig. 8. Measurement of the nearby region around the air rods. (a) Topographical information, which shows clearly the 15 air rods in the waveguide ridge. (b) Image shows the phase information, which was obtained simultaneously with the topography. Straight phase fronts with a periodicity of the wavelength of light in the material are visualized. The incoming light propagates from top to bottom. In the air rod region, a perturbation of the phase fronts is observed (dashed oval). This indicates a local change of the effective refractive index at the location where the air rods are introduced. Image size: $5 \mu \mathrm{m} \times 4 \mu \mathrm{m}$.

ferent modes propagating in the same direction interfere. If the amplitudes of the contributing modes are equal, if the modes are out of phase, and if on the two different sides of this point a different mode has the highest amplitude, a singularity with zero optical amplitude occurs [28]. Integration of the phase gradient around the position of a singularity results in an offset of the phase value by $\pm 2 \pi n$, where the integer $n$ is the so-called "topological charge" of the singularity. The "topological charge" of all phase singularities observed in the lower part of Fig. 7 is either +1 or -1 .

Fig. 8 shows a measurement of only the air rod region. Simultaneously, the topographical image [Fig. 8(a)] and the phase information [Fig. 8(b)] were detected, while light of $\lambda_{0}=632.8 \mathrm{~nm}$ was coupled into the structure. In front of the air rods (top), the straight lines corresponding to phase fronts of the incoming plane wave are visible. Inside the periodic array, a distortion in the phase fronts occurs (in the region in the dashed oval). Careful investigation of Fig. 7 shows the same distortions. This distortion from a straight wave front at the position of the air rods indicates a local change of the refractive index of the material. We find that the wavelength of light inside the periodic structure is $411(4) \mathrm{nm}$, whereas the supported mode of the waveguide structure (in front of and beyond the air rod array) had a wavelength of 439 (4) nm (Fig. 7). Thus, a shortening of wavelength by $6 \%$ takes place inside the air rod region. After $4 \mu \mathrm{m}$, a shift of almost $200 \mathrm{~nm}$ between phase fronts of light inside the air rod region in relation to phase fronts of light that propagates left and right side along the air rods is present. This shortening in wavelength is surprising. It implies an increase in the effective refractive index, whereas a lower effective index of refraction was expected due to the introduction of the air rods. Until now, no obvious explanation of this wavelength shortening is found. Due to the periodic structure, the dispersion relation can show regions with increased refractive index. We suggest that due to anomalous dispersion of light inside the array, a shortening in wavelength takes place. To understand and confirm this assumption, further 3-D calculations are needed.

To analyze the complex phase pattern in more detail, we considered the product of the measurements of the amplitude and
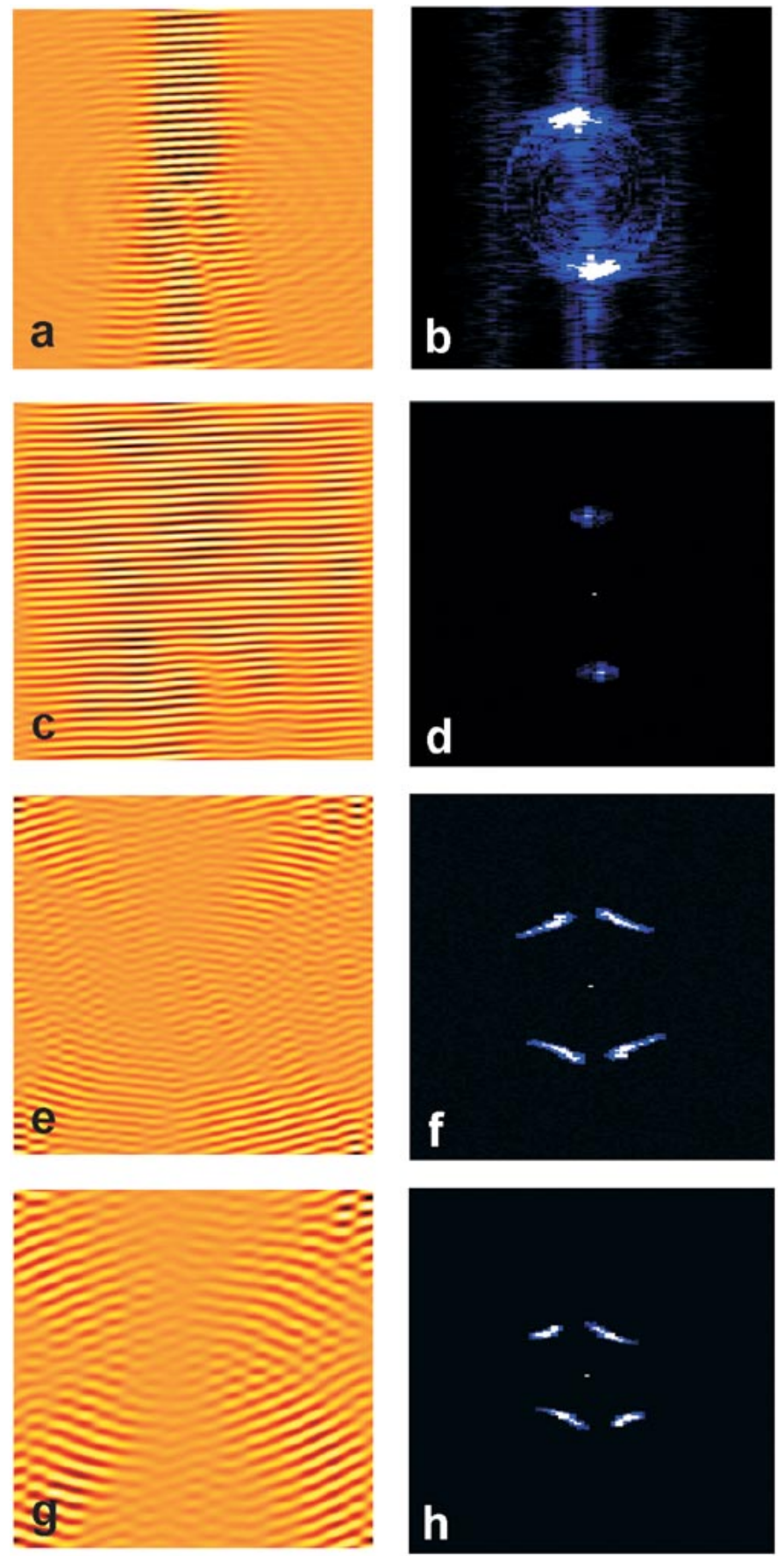

Fig. 9. Two-dimensional Fourier transformation analysis of the original measurement, which consists of the cosine of the phase times the amplitude of light $(\mathrm{A} \cdot \cos \phi)$. Fig. 9(a) shows the original data: A $\cos \phi$. Fig. 9(b) shows the spatial frequencies as given by the amplitude of the 2-D Fourier transformation of Fig. 9(a). For a detailed analysis, we have separated the features found in the Fourier transform of Fig. 9(b). The three most dominant features are shown in Fig. 9(d), (f), and (h). To see the contributing details clearly, different intensity normalization was used for Fig. 9(b), (d), (f), and (h). Investigating those components by Fourier back transformation untangles in all three cases plane waves, which propagate under different angles. Fig. 9(c), (e), and (g) correspond to 9(d), (f), and (h), respectively. Fig. 9(c) shows plane waves under an angle of $0^{\circ}$, which represent the incoming plane waves propagating through the structure. Fig. 9(e) reveals plane waves propagating under an angle of $15^{\circ}$, and Fig. $9(\mathrm{~g})$ depicts plane waves propagating under an angle of $21^{\circ}$. The image sizes of Fig. 9(a), (c), (e), and (g) are $9.33 \mu \mathrm{m} \times 17.23 \mu \mathrm{m}$. Fig. 9(b), (d), (f), and (h) show an area of $10.7 \mu \mathrm{m}^{-1} \times 11.6 \mu \mathrm{m}^{-1}$.

the cosine of the phase, shown in Fig. 9(a). Interference between incoming light and circularly scattered light is clearly seen. Fur- 
thermore, the intensity is mainly confined in the waveguide. Beyond the 15 air rods, two beams scattering out of the waveguide are detected. In between these two beams, clearly an increasing amplitude due to the recovery of light to the waveguide mode is observed.

In Fig. 9(b), the intensity of the 2-D Fourier transform of Fig. 9(a) is shown. Obviously, two important spatial frequency regions are pronounced, which represent the directions upwards and downwards along the waveguide axis in Fig. 9(a). Two concentric rings can be seen in Fig. 9(b). To investigate the details, we use Fourier filtering [40]. Different areas in the frequency domain are selected as a basis to reconstruct the Fourier filtered image. In this way, the complex Fourier pattern of Fig. 9(b) is split into three important contributions, shown in Fig. 9(d), (f), and (h). The first component contributing to Fig. 9(a) is a plane wave [Fig. 9(c) and (d)]. Fig. 9(d) shows the frequency image produced by Fourier filtering of Fig. 9(b). Back transformation of Fig. 9(d) was performed to obtain Fig. 9(c), which reveals a plane wave over the whole image. Using the periodicity of a plane wave $(439(4) \mathrm{nm}$ ) from the peaks in the spatial frequency image [Fig.9(b)], an effective refractive index of 1.440 is found. In Fig. 9(f) and (h), additional strong features out of the Fourier image were selected. The back transformation images [Fig. 9(e) and $(\mathrm{g})]$ show that these features represent plane waves propagating under discrete angles away from the center of the image. We find for the waves shown in Fig. 9(e) an angle of $15^{\circ}$ compared with the top-down propagation direction. From the spatial frequency found in Fig. 9(f), we calculate an effective refractive index of these waves of 1.20 (corresponding to a wavelength $\left.\lambda_{\text {mode }}=527 \mathrm{~nm}\right)$. Fig. $9(\mathrm{~g})$ shows plane waves of a refractive index of 0.90 (corresponding to a wavelength $\lambda_{\text {mode }}=703 \mathrm{~nm}$ ), which propagate under an angle of $21.5^{\circ}$. Comparing the refractive indexes found for the plane waves with the refractive indexes of leaky modes of the slab gives a reasonable match (some leaky slab modes: $\mathrm{TE}_{07}: \mathrm{n}_{\mathrm{eff}}=1.233, \mathrm{TE}_{10}: \mathrm{n}_{\mathrm{eff}}=0.980$ ).

\section{CONCLUSION}

We have shown the use of a phase-sensitive PSTM to investigate $3-\mathrm{D}$ photonic structures with $1-\mathrm{D}$ corrugations. Our technique visualizes both the amplitude and the phase of local fields inside the structures. In this way, local investigation of structures becomes possible on a subwavelength scale. The effective refractive index, as well as losses, can be determined at any position for any structure and without any model-dependent assumptions.

Investigations on two different integrated optical structures (15 air rods and 15 slits milled into the ridge of a conventional waveguide) were performed. From the optical intensity distribution measurement, we found a wavelength-dependent exponential decay rate of light inside the periodic structures. A faster decay is found for shorter wavelength. This wavelength dependence is therefore for a large fraction attributable to scattering processes, which are stronger for shorter wavelength. The scattering behavior of the two structures has been found to differ. For the air rod array, we find strong scattering into leaky modes of the waveguide that form a cylindrical wave around the corrugation region. This leads to a change in modulation depth of the standing wave close to the air rod array. The slit structure scatters more efficiently into the $\mathrm{SiO}_{2}$ buffer layer, which leads to an unexpected interference pattern behind the slits. Furthermore, we find a wavelength-dependent recovery after the slit array. Simulations confirm that, at the first few slits, much light is scattered into the $\mathrm{SiO}_{2}$ layer, which is subsequently reflected at the $\mathrm{SiO}_{2}-\mathrm{Si}$ interface. As a result, a nonperiodic interference pattern is observed behind the slit structure. Investigation of the phase evolution of light scattered around the 15 air rods reveals circularly scattered waves with their origin in the air rod region. Due to interference between this circularly scattered waves and light propagating in the structure, a network of phase jumps and phase singularities is built. Local observations of the phase information around the air rods show the change of the effective refractive index of the waveguide.

Such detailed analysis of light in and around subwavelength structures can only be obtained with a near-field setup. We found information about light scattering, which would have remained hidden by using far-field methods. We anticipate that our technique will allow the mapping of wave functions of local photon states inside 1-D or 2-D photonic crystals.

\section{ACKNOWLEDGMENT}

The authors would like to thank the Ligthwave Device group at the University of Twente for their collaboration.

\section{REFERENCES}

[1] T. F. Krauss, R. M. De la Rue, and S. Brand, "Two-dimensional photonic-bandgap structures operating at near infrared wavelengths," $\mathrm{Na}$ ture, vol. 383, pp. 699-702, Oct. 1996.

[2] J. E. G. J. Wijnhoven and W. L. Vos, "Preparation of photonic crystals made of air spheres in titania," Science, vol. 281, pp. 802-804, Aug. 1998.

[3] S. Noda, K. Tomoda, N. Yamamoto, and A. Chutinan, "Full three-dimensional photonic bandgap crystals at near-infrared wavelength," Science, vol. 289, pp. 604-606, July 2000.

[4] C. M. Soukoulis, Photonic Crystals and Light Localization in the 21st Century. Dordrecht/Boston/London: Kluwer, 2000.

[5] W. L. Vos, R. Sprik, A. van Blaaderen, A. Imhof, A. Lagendijk, and G. H. Wegdam, "Strong effects of photonic band structures on the diffraction of colloidal crystals," Phys. Rev. B, vol. 53, pp. 16231-16235, Jun. 1996.

[6] T. Yoshie, J. Vučković, A. Scherer, H. Chen, and D. Deppe, "High quality two-dimensional photonic crystal slab cavities," Appl. Phys. Lett., vol. 79, pp. 4289-4291, Dec. 2001.

[7] Y. Sugimoto, N. Ikeda, N. Carlsson, K. Asakawa, N. Kawai, and K. Inoue, "Light-propagation characteristics of Y-branch defect waveguides in AlGaAs-based air-bridge-type two-dimensional photonic crystal slabs," Opt. Lett., vol. 27, pp. 388-390, Mar. 2002.

[8] M. Lončar, T. Doll, J. Vučković, and A. Scherer, "Design and fabrication of silicon photonic crystal optical waveguides," J. Lightwave Technol., vol. 18, pp. 1402-1411, Oct. 2000.

[9] E. Yablonovitch, "Photonic crystals: Semiconductors of light," Sci. Amer, vol. 285, pp. 46-54, Dec. 2001

[10] Y. Sugimoto, N. Ikeda, N. Carlsson, K. Asakawa, N. Kawai, and K. Inoue, "Fabrication and characterization of different types of two-dimensional AlGaAs photonic crystal slabs," J. Appl. Phys., vol. 91, pp. 922-929, Feb. 2002.

[11] Y. A. Vlasov, X.-Z. Bo, J. C. Sturm, and D. J. Norris, "On-chip natural assembly of silicon photonic bandgap crystals," Nature, vol. 414, pp. 289-293, Nov. 2001

[12] A. Blanco, E. Chomski, S. Grabtchak, M. Ibisate, S. John, S. W.St. W. Leonard, C. Lopez, F. Meseguer, H. Miguez, J. P. Mondia, G. A. Ozin, O. Toader, and H. M. van Driel, "Large-scale synthesis of a silicon photonic crystal with a complete three-dimensional bandgap near 1.5 micrometers," Nature, vol. 405, pp. 437-440, May 2000.

[13] M. Notomi, K. Yamada, A. Shinya, J. Takahashi, C. Takahashi, and I. Yokohama, "Extremely large group-velocity dispersion of line-defect waveguides in photonic crystal slabs," Phys. Rev. Lett., vol. 87, p. 253902 , Dec. 2001. 
[14] J. Moosburger, M. Kamp, A. Forchel, S. Olivier, H. Benisty, C Weisbuch, and U. Oesterle, "Enhanced transmission through photonic crystal-based bent waveguides by bend engineering," Appl. Phys. Lett., vol. 79, pp. 3579-3581, Nov. 2001.

[15] J. Moosburger, M. Kamp, A. Forchel, U. Oesterle, and R. Houdré, "Transmission spectroscopy of photonic crystal based waveguides with resonant cavities," J. Appl. Phys., vol. 91, pp. 4791-4794, Apr. 2002.

[16] O. Painter and K. Srinivasan, "Polarization properties of dipolelike defect modes in photonic crystal nanocavities," Opt. Lett., vol. 27, pp. 339-341, Mar. 2002.

[17] N. F. van Hulst, F. B. Segerink, F. Achten, and B. Bölger, "Evanescentfield optical microscopy: Effects of polarization, tip shape and radiative waves," Ultramicroscopy, vol. 42-44, pp. 416-421, 1992.

[18] R. Quidant, J. C. Weeber, A. Dereux, D. Peyrade, G. Colas des Francs, C.Ch. Girard, and Y. Chen, "Addressing and imaging high optical index dielectric ridges in the optical near field," Phys. Rev. E, vol. 64, p. 066607 , Nov. 2001.

[19] D. Mulin, M. Spajer, D. Courjon, F. Carcenac, and Y. Chen, "Near field probing control of optical propagation in bidimensional guiding mesostructures," J. Appl. Phys., vol. 87, pp. 534-537, Jan. 2000.

[20] P. L. Phillips, J. C. Knight, B. J. Mangan, P. S. J. Russell, M. D. B. Charlton, and G. J. Parker, "Near-field optical microscopy of thin photonic crystal films," J. Appl. Phys., vol. 85, pp. 6337-6342, May 1999.

[21] D. Gérard, L. Berguiga, F. de Fornel, L. Salomon, C. Seassal, X. Letartre, P. Rojo-Romeo, and P. Viktorovitch, "Near-field probing of active photonic-crystal structures," Opt. Lett., vol. 27, pp. 173-175, Feb. 2002.

[22] G. H. Vander Rhodes, B. B. Goldberg, M. S. Unlü, S. T. Chu, W. Pan, T Kaneko, Y. Kokobun, and B. E. Little, "Measurement of internal spatial modes and local propagation properties in optical waveguides," Appl. Phys. Lett., vol. 75, pp. 2368-2370, Oct. 1999.

[23] G. H. Vander Rhodes, M. S. Ünlü, B. B. Goldberg, J. M. Pomeroy, and T. F. Krauss, "Characterization of waveguide microcavities using high-resolution transmission spectroscopy and near-field scanning optical microscopy," Inst. Elect. Eng. Proc.-J.: Optoelectron, vol. 145, pp. 379-382, Dec. 1998.

[24] J. R. Krenn, A. Dereux, J. C. Weeber, E. Bourillot, Y. Lacroute, J. P. Gourdonnet, G. Schider, W. Gotschy, A. Leitner, F. R. Aussenegg, and C. Girard, "Squeezing the optical near-field zone by plasmon coupling of metallic nanoparticles," Phys. Rev. Lett., vol. 82, pp. 2590-2593, Mar. 1999.

[25] P. Kramper, A. Birner, M. Agio, C. M. Soukoulis, F. Müller, U. Gösele, J. Mlynek, and V. Sandoghdar, "Direct spectroscopy of a deep two-dimensional photonic crystal microresonator," Phys. Rev. B, vol. 64, p. 233 102, Nov. 2001.

[26] A. L. Campillo, J. W. P. Hsu, and G. W. Bryant, "Local imaging of photonic structures: image contrast from impedance mismatch," Opt. Lett., vol. 27, pp. 415-417, Mar. 2002

[27] M. L. M. Balistreri, J. P. Korterik, G. J. Veldhuis, L. Kuipers, and N. F van Hulst, "Quantitative photon tunelling and shear-force microscopy of planar waveguide splitters and mixers," J. Appl. Phys., vol. 89, pp. 3307-3314, Mar. 2001

[28] M. L. M. Balistreri, J. P. Korterik, L. Kuipers, and N. F. van Hulst, "Local observation of phase singularities in optical fields in waveguide structures," Phys. Rev. Lett., vol. 85, pp. 294-297, Jul. 2000.

[29] P. L. Phillips, J. C. Knight, J. M. Pottage, G. Kakarantzas, and P. St. Russell, "Direct measurement of optical phase in the near field," Appl. Phys. Lett., vol. 76, pp. 541-543, Jan. 2000.

[30] M. L. M. Balistreri, J. P. Korterik, L. Kuipers, and N. F. van Hulst, "Phase mapping of optical fields in integrated optical waveguide structures," $J$. Lightwave Technol., vol. 19, pp. 1169-1176, Aug. 2001.
[31] A. Nesci, R. Dändliker, M. Salt, and H. P. Herzig, "Optical near-field phase singularities produced by microstructues," in Proc. SPIE, San Diego, CA, Aug. 2001, pp. 68-77.

[32] K. Karrai and R. D. Grober, "Piezoelectric tip-sample distance control for near field optical microscopes," Appl. Phys. Lett., vol. 66, pp. 1842-1844, Apr. 1995.

[33] M. Vaez-Iravani and R. Toledo-Crow, "Phase contrast and amplitude pseudoheterodyne interference near field scanning optical microscopy," Appl. Phys. Lett., vol. 62, pp. 1044-1046, Mar. 1993.

[34] J. A. Veerman, A. M. Otter, L. Kuipers, and N. F. van Hulst, "High definition aperture probes for near-field optical microscopy fabricated by focused ion beam milling," Appl. Phys. Lett., vol. 72, pp. 3115-3117, Jun. 1998.

[35] C. Peeters, E. Flück, A. M. Otter, M. L. M. Balistreri, J. P. Korterik, L. Kuipers, and N. F. van Hulst, "Photon scanning tunneling microscopy of tailor-made photonic structures," Appl. Phys. Lett., vol. 77, pp. 142-144, July 2000

[36] S. Fan, I. Appelbaum, and J. D. Joannopoulos, "Near-field scanning optical microscopy as a simultaneous probe of fields and band structure of photonic crystals: a computational study," Appl. Phys. Lett., vol. 75, pp. 3461-3463, Nov. 1999

[37] T. Tamir, Integrated Optics. Berlin, Germany: Springer, 1975.

[38] M. Lohmeyer and R. Stoffer, "Integrated optical cross strip polarizer concept," Opt. Quantum Electron., vol. 33, pp. 413-431, Apr. 2001.

[39] M. Lohmeyer, "Mode expansion modeling of rectangular integrated optical microresonators," Opt. Quantum Electron., vol. 34, pp. 541-557, June 2002.

[40] M. L. M. Balistreri, J. P. Korterik, L. Kuipers, and N. F. van Hulst, "Visualization of modes transformation in a planar waveguide splitter by near-field optical imaging," Appl. Phys. Lett., vol. 79, pp. 910-912, Aug. 2001.

E. Flück, photograph and biography not available at the time of publication.

M. Hammer, photograph and biography not available at the time of publication

A. M. Otter, photograph and biography not available at the time of publication.

J. P. Korterik, photograph and biography not available at the time of publication.

L. Kuipers, photograph and biography not available at the time of publication.

N. F. van Hulst, photograph and biography not available at the time of publication. 